\title{
Antioxidant capacity and polyphenol content of the encapsulated pulpe of Opuntia ficus yellow variety
}

\begin{abstract}
The yellow prickly pear (Opuntia ficus-indica) is a seasonal fruit of the Huancavelica region, which is used very little, despite being a source of phenolic compounds and natural antioxidants, which is why encapsulated yellow prickly pear pulp was developed using maltodextrin, in such a way that its functional properties are preserved. A $2^{k}$ factorial design was used whose independent variables were the encapsulating agent ratio $(10 \%$ and $20 \%)$ and the inlet air temperature $\left(120^{\circ} \mathrm{C}\right.$ and $140{ }^{\circ} \mathrm{C}$ ), and the dependent variables were: the polyphenol content totals (Folin - Ciocalteau method), antioxidant activity (2,2-diphenyl-1-picrylhydracil free radical method), moisture (gravimetric method), yield and colorimetry parameters $(a, b, L)$ to measure encapsulation efficiency. The maximum total polyphenol content and antioxidant activity reached were 231.16 $\pm 0.406 \mathrm{mg}$ gallic acid equivalent / $100 \mathrm{~g}$ and $53.86 \pm 2.85 \%$ inhibition, respectively: for an encapsulating agent ratio of $10 \%$ and inlet air temperature $120{ }^{\circ} \mathrm{C}$. With these values of the independent variables, moisture, and yield values of $6.49 \pm 0.06 \%$ and $7.45 \pm 0.15 \%$, respectively, were obtained. The stability of the color of the encapsulation, as well as its functional properties are achieved at a ratio of $10 \%$ and inlet temperature of drying air at $120^{\circ} \mathrm{C}$.
\end{abstract}

Keywords - antioxidant activity, functional foods, phenolic compounds, encapsulation, Opuntia ficus-indica, maltodextrin.

\section{INTRODUCCIÓN}

Este cactus es de origen mexicano, pero ahora está ampliamente distribuido en regiones áridas y semiáridas del Nuevo y Viejo Mundo. Produce frutas dulces comestibles, ricas en nutrientes y sus tallos espatulados (llamados cladodios) son ingredientes de la cocina mexicana [1]. Estos frutos varían en colores que van desde blanco, amarillo, naranja, hasta el rojo y morado [2]. En el Perú, las tunas se producen en zonas andinas muy importantes como: Ayacucho (20,25\%), Huancavelica (16.65\%), Arequipa (14.84\%), Lima (14,02\%) y Apurímac (8,24\%). Este cultivo reduce la desertificación y la erosión del suelo, consume $\mathrm{C} 02$, y disminuye la contaminación del aire [3]. La provincia de Churcampa produce la tuna amarilla, sin darle un valor agregado y con mucho desperdicio por falta de mercado.

La tuna amarilla es rica en betalaínas, particularmente betaxantinas (amarillo-naranja), y en menor cantidad en betacianinas (rojo-violeta) [4]. Son una fuente de colorantes alimentarios amarillos naturales [2], [5]. Este pigmento es una alternativa al amarillo-naranja sintético como Tartrazine (E 102) y Sunset Yellow (E110) cuyo uso es restringido [6] porque podrían inducir hiperactividad en la infancia [7], dermatitis atópica, urticaria y angioedema humano [8].
Además de las betalaínas, las tunas poseen un alto contenido fenólico, siendo los más importantes los derivados flavonoides [9]. Muestran interesantes beneficios para la salud en términos de efectos antiinflamatorios y quimiopreventivos [10]. Con respecto a los ácidos fenólicos individuales, se han identificado ácidos hidroxibenzoico, fusídico y cafeico, así como derivados del ácido ferúlico y fusídico en los jugos de tuna [11].

Se han identificado 37 metabolitos secundarios mediante HPLC-MS / MS, en el polisacárido de la pulpa de frutos y cladodios. Todos los extractos de tuna exhibieron actividades antioxidantes sustanciales in vitro y potencial neuroprotector en la enfermedad de Alzheimer inducida por cloruro de aluminio. También disminuyeron significativamente los niveles cerebrales elevados de citocinas proinflamatorias, aumento de citocinas antiinflamatorias y neurotransmisores monoamínicos en comparación con el grupo de control positivo [1].

La estabilidad de estos compuesto bioactivos es un parámetro importante cuando se utilizan estos pigmentos como antioxidantes y colorantes en alimentos. El color, los compuestos fenólicos y la actividad antioxidante se ven afectados por el $\mathrm{pH}$, la actividad del agua, la exposición a la luz, el oxígeno, los metales, los antioxidantes, la temperatura y la actividad enzimática, siendo la temperatura el factor más decisivo [12], [13]. Por lo tanto, la estabilización los compuestos bioactivos de la pulpa se puede mejorar usando tecnologías de microencapsulación, como spray-drying o secado por aspersión, que es el método de encapsulación más utilizado debido a su costo relativamente bajo y a la disponibilidad de equipos [14].

Mediante secado por aspersión se ha encapsulado betaxantina con maltodextrina [15], [16]. También se ha encapsulado betaxantinas junto con betacianinas por aspersión usando maltodextrina e inulina [17], [18], almidón modificado [19], y mucílagos de cladodios de tuna [20]. Finalmente se ha encapsulado betaxantinas amarillas y se han optimizado las temperaturas de secado por aspersión [21].

En este trabajo de investigación se usado como agente encapsulante a la maltodextrina por su bajo costo y disponibilidad en el mercado, y se propuso obtener una relación pulpa: agente encapsulante (P: EA) y temperaturas de entrada adecuadas para que la encapsulación de pulpa de Opuntia ficus-indica variedad amarilla retenga su color, capacidad antioxidante y contenido de polifenoles. 


\section{MATERIALES Y MÉTODOS}

A. Diseño experimental y análisis de datos

TABLA I

DISEÑO FACTORIAL COMPLETO $2^{2}$

\begin{tabular}{clc}
\multicolumn{3}{c}{ DiSEÑO FACTORIAL COMPLETO 2} \\
\hline $\begin{array}{c}\text { Temperatura } \\
\left({ }^{\circ} \mathrm{C}\right)\end{array}$ & \multicolumn{2}{c}{ Ratio encapsulante/pulpa } \\
\cline { 2 - 3 } 120 & $10 \%$ & $20 \%$ \\
\hline \multirow{2}{*}{120} & $\mathrm{R}_{111}$ & $\mathrm{R}_{212}$ \\
& $\mathrm{R}_{112}$ & $\mathrm{R}_{213}$ \\
& $\mathrm{R}_{113}$ & $\mathrm{R}_{221}$ \\
& $\mathrm{R}_{121}$ & $\mathrm{R}_{222}$ \\
& $\mathrm{R}_{122}$ & $\mathrm{R}_{223}$ \\
\hline
\end{tabular}

Se aplicó un Diseño Factorial Completo $2^{2}$ y los resultados se sometieron a un análisis de varianza (Tabla I).

\section{B. Obtención encapsulado de pulpa de tuna}

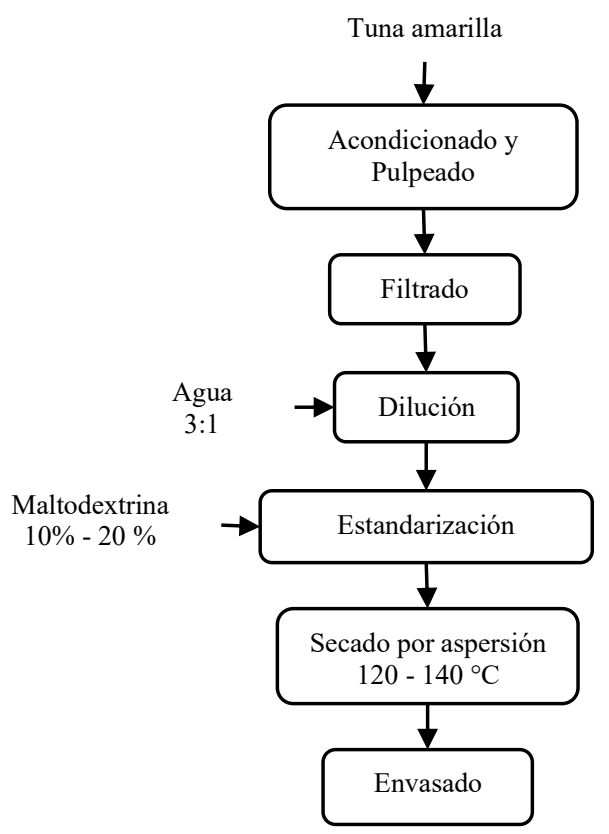

Fig. 1 Diagrama de flujo de encapsulado de pulpa de tuna.

Se usaron $50 \mathrm{~kg}$ de tuna amarilla de Churcampa, Huancavelica, separándose tunas en buen estado, que mejoren la calidad del producto final. Se acondicionaron los frutos lavando con agua potable y desinfectaron con agua clorada a 100 ppm. Luego se obtuvo la pulpa y se filtró con un tamiz de 0,5 mm. La pulpa filtrada se diluyó a 1 volumen con 3 de agua. En la estandarización, se manipuló la primera variable, ratio encapsulante: pulpa (REP), el encapsulante se adicionó a $10 \%$ y $20 \%$ en función al peso de la dilución de pulpa con agua, y se mezcló a agitación constante durante 5 min. Las soluciones resultantes fueron alimentadas en un Mini Spray Dryer B - 290 marca Buchi. En el secado por aspersión se manipuló la segunda variable independiente, la temperatura de aire de entrada (TA), en un rango de 120 a $140 \pm 5^{\circ} \mathrm{C}$. Otras variables como el flujo de aire y velocidad de alimentación se mantuvieron constantes a valores de 473
$\mathrm{L} / \mathrm{h}$ y $10 \mathrm{~mL} / \mathrm{min}$, respectivamente. El encapsulado final fue envasado en bolsa de polipropileno y se almacenó protegido de la exposición a la luz a $20{ }^{\circ} \mathrm{C}$ para su posterior análisis.

\section{Contenido de polifenoles totales (CPT)}

Se pesó $200 \mathrm{mg}$ de encapsulado y se dispersó en $1 \mathrm{~mL}$ de acetonitrilo y $1 \mathrm{~mL}$ de metanol : ácido acético : agua $(50: 8: 42 \mathrm{v} / \mathrm{v} / \mathrm{v})$. Esta dispersión se agitó con un vortex (1 min), se ultrasonicó dos veces por $20 \mathrm{~min}$, se centrifugó a $112,000 \mathrm{~g}$ por $5 \mathrm{~min}$, y se filtró (0.22 mm Millipore filter). El contenido de polifenoles se cuantificó por el método de Folin-Ciocalteau [22].

\section{Actividad antioxidante (AA)}

Se mezclaron $0,1 \mathrm{~mL}$ de muestras con $0,9 \mathrm{~mL}$ de 100 $\mathrm{mM}$ Trise- $\mathrm{HCl}$ buffer $(\mathrm{pH} 7,4)$ a los cuales se le añadieron 1 $\mathrm{mL}$ de DPPH (2,2-diphenyl-1-picrylhydracil) $(0,500 \mathrm{mM}$ en etanol). La muestra control fue preparada de manera similar añadiendo $0,1 \mathrm{~mL}$ de agua en lugar de la muestra. Las mezclas fueron agitadas vigorosamente y se dejaron por 30 min. La absorbancia de la solución resultante fue medida a $517 \mathrm{~nm}$ por un espectrofotómetro UV-vis. La mezcla de reacción sin DPPH se utilizó para la corrección de fondo. La ecuación se aplicó para evaluar la actividad antioxidante [23]:

$$
A A=\left[1-A_{\text {muestra }} / A_{\text {eontrol }}\right] x 100 \text { (1) }
$$

Donde: $\mathrm{A}_{\text {muestra }}$ es la absorbancia del microencapsulado a $517 \mathrm{~nm}$ y $\mathrm{A}_{\text {control }}$ es la absorbancia de la muestra control a $517 \mathrm{~nm}$.

\section{E. Color}

El color de la muestra ( $\mathrm{L}, \mathrm{a}^{*}, \mathrm{y} \mathrm{b}^{*}$ ) fue medida usando un Equipo MINOLTA CR-200b.

\section{F. Humedad (\%H)}

Se usó el método de la AOAC 925.4072, basada en la pérdida de peso que sufre la muestra por calentamiento hasta obtener peso constante.

\section{G. Rendimiento ( $\eta$ )}

El rendimiento del microencapsulado se calculó con la siguiente fórmula:

$$
\eta=\frac{P_{m e}}{P_{t a}} x 100(2)
$$

Dónde: $\mathrm{P}_{\mathrm{mc}}=$ Peso en gramos del microencapsulado, $\mathrm{P}_{\mathrm{ta}}=$ Peso en gramos de la pulpa de tuna amarilla diluida que entra al atomizador.

\section{H. Eficiencia de Encapsulación (EE)}

El porcentaje de retención o eficiencia de encapsulación se calculó con la siguiente fórmula:

$$
E E=\frac{P_{E}}{P_{F}} x 100
$$


Dónde: $\mathrm{P}_{\mathrm{E}}=$ concentración de compuesto bioactivo en el encapsulado en base seca. $\mathrm{P}_{\mathrm{F}}=$ concentración de compuesto bioactivo en el producto fresco en base seca.

\section{RESULTADOS Y DISCUSIONES}

\section{A. Análisis estadístico}

TABLA II

\begin{tabular}{|c|c|c|c|}
\hline \multirow{3}{*}{$\begin{array}{l}\text { Variables } \\
\text { de } \\
\text { respuesta }\end{array}$} & \multicolumn{3}{|c|}{ Fuente de variación } \\
\hline & REP & TA & REP - TA \\
\hline & \multicolumn{3}{|c|}{$\mathrm{p}$} \\
\hline CPT & $0,000 *$ & $0,000^{*}$ & $0,000^{*}$ \\
\hline AA & $0,000 *$ & $0,000^{*}$ & $0,000 *$ \\
\hline $\mathrm{L}$ & $0,007 *$ & $0,018^{*}$ & $0,014^{*}$ \\
\hline$a^{*}$ & $0,000^{*}$ & $0,000^{*}$ & $0,000^{*}$ \\
\hline $\mathrm{b}^{*}$ & $0,000^{*}$ & $0,000^{*}$ & $0,000^{*}$ \\
\hline$\% \mathrm{H}$ & $0,000 *$ & $0,000^{*}$ & 0,812 \\
\hline$\eta$ & $0,000^{*}$ & $0,000^{*}$ & $0,000 *$ \\
\hline
\end{tabular}

EFECTOS PRINCIPALES

\begin{tabular}{cccc}
$\begin{array}{c}\text { Variables } \\
\text { de } \\
\text { respuesta }\end{array}$ & \multicolumn{2}{c}{ Fuente de variación } & \multirow{2}{*}{$\mathrm{R}^{2}$} \\
\cline { 2 - 3 } & REP & TA & \\
\cline { 2 - 3 } CPT & - & - & $98,38 \%$ \\
AA & - & - & $99,20 \%$ \\
L & - & + & $54,84 \%$ \\
$\mathrm{a}^{*}$ & + & + & $96,91 \%$ \\
$\mathrm{~b}^{*}$ & - & + & $97,28 \%$ \\
$\% \mathrm{H}$ & - & - & $99,77 \%$ \\
$\eta$ & + & + & $99,53 \%$ \\
\hline (-): INFLUENCIA NEGATIVA; (+): INFLUENCIA POSITIVA
\end{tabular}

En la tabla II, se puede observar que, la ratio de encapsulante:pulpa (REP), la temperatura de entrada (TA) y la interacción de estos factores (REP - TA) tuvieron un efecto significativo $(\mathrm{p}<0,05)$ sobre el contenido en polifenoles totales del encapsulado de tuna amarilla (CPT). El modelo de regresión para el contenido de polifenoles totales tuvo un alto coeficiente de determinación $\left(\mathrm{R}^{2}=\right.$ $98.38 \%$ ), lo que permite predecir el contenido en polifenoles totales del encapsulado usando las variables de estudio dentro de los rangos estudiados (tabla III), además se deduce que los efectos principales tuvieron un efecto negativo en CPT. Al respecto, Ileana et al. encontraron que las condiciones de secado estudiadas (temperatura de entrada y de salida) no afectaron significativamente $(\mathrm{p}<0,05)$ la retención de los compuestos fenólicos [24].

La ratio de encapsulante, la temperatura de entrada y la interacción de estos factores tuvieron un efecto significativo $(\mathrm{p}<0,05)$ sobre la actividad antioxidante del encapsulado de tuna amarilla (tabla II). El modelo de regresión para actividad antioxidante tuvo un alto coeficiente de determinación (tabla III), y se apreció que las variables de estudio afectan negativamente la actividad antioxidante. Esto es contrario, a otro sistemas alimentarios donde reacciones de condensación entre las antocianinas y otros antioxidantes, resultando en la formación de compuestos fenólicos polimerizados que mantenían la actividad antioxidante [25]. En jugo de melón secado por aspersión se observó que todos los jugos reconstituidos mostraron menores actividades antioxidantes en comparación con el jugo fresco [26].

En cuanto a los parámetros de color $\left(\mathrm{L}, \mathrm{a}^{*}, \mathrm{~b}^{*}\right)$ se puede apreciar que existe efecto significativo del REP y la TA ( $\mathrm{p}<$ $0,05)$. Las muestras secadas con la mayor concentración del agente portador fueron más oscuras, mostrando menores valores de luminosidad (L). Además, la muestra secada a la temperatura más alta $(140 \circ \mathrm{C})$ fue la más clara. Esta muestra fue probablemente la menos susceptible a la caramelización [27], [28].

La ratio de encapsulante, la temperatura de entrada y la interacción de estos factores tuvieron un efecto significativo $(\mathrm{p}<0,05)$ sobre la humedad del encapsulado y el modelo de regresión para la humedad tuvo un alto coeficiente de determinación $\left(\mathrm{R}^{2}=99,77 \%\right)$, siendo los efectos principales negativos para la humedad. La maltodextrina es un agente encapsulante muy importante, porque forman soluciones de baja viscosidad en altas concentraciones que pueden ser secadas por pulverización de manera eficiente [29], permitiendo obtener un polvo con bajo contenido de humedad, baja higroscopicidad y alta solubilidad [27]. En este trabajo, se usó la maltodextrina y se logró una humedad baja dentro de un rango de $4,04 \%$ a $6,57 \%$.

TABLA IV

CARACTERÍSTICAS DEL ENCAPSULADO DE PULPA DE TUNA AMARILLA

\begin{tabular}{lc}
\multicolumn{2}{c}{ Característica } \\
\hline \multicolumn{2}{c}{ Valor } \\
\hline Polifenoles totales (mg ác. gálico/100g) & $231,16 \pm 0,406$ \\
Capacidad Antioxidante (inhibición) & $53,86 \pm 2,85 \%$ \\
L* $^{*}$ Y REP: $\left.10 \%\right)$ & $95,53 \pm 0,19$ \\
$a^{*}$ & $-3,41 \pm 0,04$ \\
$b^{*}$ & $22,55 \pm 0,14$ \\
Humedad & $6,49 \pm 0,06 \%$ \\
Rendimiento $(\eta)$ & $7,45 \pm 0,15 \%$ \\
EE polifenoles totales & $59,65 \pm 0,91 \%$ \\
EE capacidad antioxidante & $97,90 \pm 0,45 \%$ \\
\hline
\end{tabular}

Finalmente, una ratio de $10 \%$ y temperatura de entrada de $120{ }^{\circ} \mathrm{C}$ se logra un encapsulado de pulpa de tuna amarilla con un contenido en polifenoles totales de $231.16 \mathrm{mg}$ de ácido gálico / g materia seca, una capacidad antioxidante de $53.86 \%$, humedad $6.49 \%$, rendimiento $7.45 \%$ y un polvo de color claro. Además, presenta mayor eficiencia de encapsulado (EE) en polifenoles totales $(59,65 \%)$ y capacidad antioxidante $(97,90 \%)$. Carmona et al. encontró las condiciones óptimas para la encapsulación pulpa de tuna amarilla-naranja con maltodextrina a temperatura del aire de entrada $150^{\circ} \mathrm{C}$ y una relación encapsulante pulpa de 3: 1, donde la relación pulpa: agente encapsulante tuvo más influencia que la temperatura en el rendimiento durante la encapsulación [30]. 


\section{CONCLUSIONES}

El uso de $10 \%$ de maltodextrina y $120{ }^{\circ} \mathrm{C}$ de temperatura de entrada de aire de secado para la encapsulación de la pulpa de tuna amarilla mejoraron el contenido de polifenoles y la estabilidad de la actividad antioxidante, como lo demuestra la eficiencia de encapsulación, así como el color y la humedad final del producto. Las micropartículas de tuna amarilla podrían usarse como ingredientes alimentarios para alimentos funcionales debido a su contenido de antioxidantes y propiedades colorantes. De esta manera, se daría valor agregado a esta materia prima poco valorada en la provincia de Churcampa, Huancavelica.

\section{REFERENCIAS}

[1] S. S. El-Hawary et al., "HPLC-PDA-MS/MS profiling of secondary metabolites from Opuntia ficus-indica cladode, peel and fruit pulp extracts and their antioxidant, neuroprotective effect in rats with aluminum chloride induced neurotoxicity," Saudi J. Biol. Sci., vol. 27, no. 10, pp. 2829-2838, 2020.

[2] F. C. Stintzing et al., "Color, betalain pattern, and antioxidant properties of cactus pear (Opuntia spp.) clones," J. Agric. Food Chem., vol. 53, no. 2, pp. 442-451, 2005.

[3] PNIA, "Productores de Apurímac mejoran producción y calidad de tuna con proyecto de innovación | Gobierno del Perú," 2020. https://www.gob.pe/institucion/pnia/noticias/126050-productoresde-apurimac-mejoran-produccion-y-calidad-de-tuna-conproyecto-de-innovacion (accessed Sep. 05, 2021).

[4] M. I. Khan, "Stabilization of betalains: A review," Food Chem., vol. 197, pp. 1280-1285, 2016.

[5] M. J. Cejudo-Bastante, M. Chaalal, H. Louaileche, J. Parrado, and F. J. Heredia, "Betalain profile, phenolic content, and color characterization of different parts and varieties of Opuntia ficusindica," J. Agric. Food Chem., vol. 62, no. 33, pp. 8491-8499, 2014.

[6] E. P. on F. A. and N. S. added to F. (ANS), "Reconsideration of the temporary ADI and refined exposure assessment for Sunset Yellow FCF (E 110)," EFSA J., vol. 12, no. 7, p. 3765, 2014.

[7] D. McCann et al., "Food additives and hyperactive behaviour in 3-year-old and 8/9-year-old children in the community: a randomised, double-blinded, placebo-controlled trial," Lancet, vol. 370, no. 9598, pp. 1560-1567, 2007.

[8] M. L. T. Polônio and F. Peres, "Food additive intake and health effects: public health challenges in Brazil," Cad. Saude Publica, vol. 25, no. 8, pp. 1653-1666, 2009.

[9] T. García-Cayuela, A. Gómez-Maqueo, D. Guajardo-Flores, J Welti-Chanes, and M. P. Cano, "Characterization and quantification of individual betalain and phenolic compounds in Mexican and Spanish prickly pear (Opuntia ficus-indica L. Mill) tissues: A comparative study," J. Food Compos. Anal., vol. 76, pp. 1-13, 2019.

[10] M. Antunes-Ricardo, C. Rodríguez-Rodríguez, J. A. GutiérrezUribe, E. Cepeda-Cañedo, and S. O. Serna-Saldívar, "Bioaccessibility, intestinal permeability and plasma stability of isorhamnetin glycosides from Opuntia ficus-indica (L.)," Int. J. Mol. Sci., vol. 18, no. 8, p. 1816, 2017.

[11] A. Mata, J. P. Ferreira, C. Semedo, T. Serra, C. M. M. Duarte, and M. R. Bronze, "Contribution to the characterization of Opuntia spp. juices by LC-DAD-ESI-MS/MS," Food Chem., vol. 210 , pp. 558-565, 2016.

[12] G. B. Celli and M. S.-L. Brooks, "Impact of extraction and processing conditions on betalains and comparison of properties with anthocyanins-A current review," Food Res. Int., vol. 100, pp. 501-509, 2017.

[13] C. Betancourt, M. J. Cejudo-Bastante, F. J. Heredia, and N. Hurtado, "Pigment composition and antioxidant capacity of betacyanins and betaxanthins fractions of Opuntia dillenii (Ker Gawl) Haw cactus fruit," Food Res. Int., vol. 101, pp. 173-179,
2017

P. Robert, V. Torres, P. García, C. Vergara, and C. Sáenz, "The encapsulation of purple cactus pear (Opuntia ficus-indica) pulp by using polysaccharide-proteins as encapsulating agents," $L W T$ Food Sci. Technol., vol. 60, no. 2, pp. 1039-1045, 2015.

[15] J. A. Fernández-López, M. J. Roca, J. M. Angosto, and J. M. Obón, "Betaxanthin-rich extract from cactus pear fruits as yellow water-soluble colorant with potential application in foods," Plant Foods Hum. Nutr., vol. 73, no. 2, pp. 146-153, 2018.

[16] M. C. Otálora, J. G. Carriazo, C. Osorio, and M. A. Nazareno, "Encapsulation of cactus (Opuntia megacantha) betaxanthins by ionic gelation and spray drying: A comparative study," Food Res. Int., vol. 111, pp. 423-430, 2018.

[17] C. Saénz, S. Tapia, J. Chávez, and P. Robert, "Microencapsulation by spray drying of bioactive compounds from cactus pear (Opuntia ficus-indica)," Food Chem., vol. 114, no. 2, pp. 616-622, 2009.

[18] K. Ravichandran et al., "Effects of different encapsulation agents and drying process on stability of betalains extract," J. Food Sci. Technol., vol. 51, no. 9, pp. 2216-2221, 2014.

[19] C. Vergara, J. Saavedra, C. Sáenz, P. García, and P. Robert, "Microencapsulation of pulp and ultrafiltered cactus pear (Opuntia ficus-indica) extracts and betanin stability during storage," Food Chem., vol. 157, pp. 246-251, 2014.

[20] D. Soto-Castro, M. Chávez-Gutiérrez, F. León-Martínez, P. I. Santiago-García, Araceli Aragón-Lucero, and F. AntonioAntonio, "Spray drying microencapsulation of betalain rich extracts from Escontria chiotilla and Stenocereus queretaroensis fruits using cactus mucilage," Food Chem., vol. 272, pp. 715722, 2019.

[21] F. Gandia-Herrero, M. Jimenez-Atienzar, J. Cabanes, F. GarciaCarmona, and J. Escribano, "Stabilization of the bioactive pigment of Opuntia fruits through maltodextrin encapsulation," $J$. Agric. Food Chem., vol. 58, no. 19, pp. 10646-10652, 2010.

[22] V. L. Singleton and J. A. Rossi, "Colorimetry of Total Phenolics with Phosphomolybdic-Phosphotungstic Acid Reagents," Am. J. Enol. Vitic., vol. 16, no. 3, pp. 144-158, 1965, Accessed: Jan. 17, $2020 . \quad$ [Online]. Available: https://www.ajevonline.org/content/16/3/144.

[23] M. Çam, Y. Hışıl, and G. Durmaz, "Classification of eight pomegranate juices based on antioxidant capacity measured by four methods," Food Chem., vol. 112, no. 3, pp. 721-726, 2009.

[24] K. Ileana, T. Madrid, G. Ismael, O. Revilla, T. Guadalupe, and G. Velázquez, "Encapsulación por secado por aspersión de compuestos fenólicos de la cáscara de tuna morada (Opuntia ficus-indica) utilizando goma arábiga y maltodextrina como agente encapsulante," 2016.

[25] C. B. Casati, R. Baeza, V. Sanchez, A. Catalano, P. López, and M. C. Zamora, "Thermal degradation kinetics of monomeric anthocyanins, colour changes and storage effect in elderberry juices," J. Berry Res., vol. 5, no. 1, pp. 29-39, 2015.

[26] L. Wang, A. Clardy, D. Hui, and Y. Wu, "Physiochemical properties of encapsulated bitter melon juice using spray drying," Bioact. Carbohydrates Diet. Fibre, p. 100278, 2021.

[27] V. B. de Souza, M. Thomazini, J. C. de Carvalho Balieiro, and C. S. Fávaro-Trindade, "Effect of spray drying on the physicochemical properties and color stability of the powdered pigment obtained from vinification byproducts of the Bordo grape (Vitis labrusca)," Food Bioprod. Process., vol. 93, pp. 3950, 2015.

[28] R. V Tonon, A. F. Baroni, C. Brabet, O. Gibert, D. Pallet, and M. D. Hubinger, "Water sorption and glass transition temperature of spray dried açai (Euterpe oleracea Mart.) juice," J. Food Eng., vol. 94, no. 3-4, pp. 215-221, 2009.

[29] V. B. de Souza et al., "Functional properties and stability of spray-dried pigments from Bordo grape (Vitis labrusca) winemaking pomace," Food Chem., vol. 164, pp. 380-386, 2014.

[30] J. C. Carmona, P. Robert, C. Vergara, and C. Sáenz, "Microparticles of yellow-orange cactus pear pulp (Opuntia ficus-indica) with cladode mucilage and maltodextrin as a food coloring in yogurt," $L W T$, vol. 138, p. 110672, 2021. 